\title{
A BDDC METHOD FOR MORTAR DISCRETIZATIONS USING A TRANSFORMATION OF BASIS*
}

\author{
HYEA HYUN KIM ${ }^{\dagger}$, MAKSYMILIAN DRYJA ${ }^{\ddagger}$, AND OLOF B. WIDLUND ${ }^{\S}$
}

\begin{abstract}
A BDDC (balancing domain decomposition by constraints) method is developed for elliptic equations, with discontinuous coefficients, discretized by mortar finite element methods for geometrically nonconforming partitions in both two and three space dimensions. The coarse component of the preconditioner is defined in terms of one mortar constraint for each edge/face, which is the intersection of the boundaries of a pair of subdomains. A condition number bound of the form $C \max _{i}\left\{\left(1+\log \left(H_{i} / h_{i}\right)\right)^{2}\right\}$ is established under certain assumptions on the geometrically nonconforming subdomain partition in the three-dimensional case. Here $H_{i}$ and $h_{i}$ are the subdomain diameters and the mesh sizes, respectively. In the geometrically conforming case and the geometrically nonconforming cases in two dimensions, no assumptions on the subdomain partition are required. This BDDC preconditioner is also shown to be closely related to the Neumann-Dirichlet version of the FETI-DP algorithm. The results are illustrated by numerical experiments which confirm the theoretical results.
\end{abstract}

Key words. elliptic problems, finite elements, mortar methods, parallel algorithms, preconditioner, BDDC, FETI-DP, change of basis

AMS subject classifications. 65F10, 65N30, 65N55

DOI. $10.1137 / 070697859$

1. Introduction. This study concerns a scalable BDDC (balancing domain decomposition by constraints) method for solving linear systems arising from mortar finite element discretizations of elliptic problems with discontinuous coefficients. BDDC methods were first introduced by Dohrmann [5] as an alternative to and an improvement of the balancing Neumann-Neumann methods. These more recent methods use different and more flexible coarse finite element spaces which lead to sparser linear systems. Additionally, as in the dual-primal finite element tearing and interconnecting (FETI-DP) methods, all linear systems actually solved have symmetric, positive definite coefficient matrices.

The coarse basis functions are related to a relatively small set of continuity constraints, across the interface between the subdomains, which are enforced throughout the iteration. In the standard, conforming finite element case, these constraints are given in terms of common values at subdomain vertices and/or common values of averages computed over subdomain edges and/or faces. We will refer to these as primal constraints and the corresponding subspace as the primal space of displacements. We

\footnotetext{
* Received by the editors July 20, 2007; accepted for publication (in revised form) July 16, 2008; published electronically October 24, 2008.

http://www.siam.org/journals/sinum/47-1/69785.html

${ }^{\dagger}$ Department of Mathematics, Chonnam National University, Youngbong-dong, Buk-gu, Gwangju 500-757, Korea (hyeahyun@gmail.com or hkim@chonnam.ac.kr). This author's research was supported in part by the U.S. Department of Energy under contract DE-FC02-01ER25482 and in part by the Post-doctoral Fellowship Program of Korea Science and Engineering Foundation (KOSEF).

¥Department of Mathematics, Warsaw University, Banacha 2, 02-097 Warsaw, Poland (dryja@ mimuw.edu.pl). This author's research was supported in part by the Polish Science Foundation under grant NN201006933.

${ }^{\S}$ Department of Mathematics, Courant Institute of Mathematical Sciences, 251 Mercer Street, New York, NY 10012 (widlund@cs.nyu.edu, http://cs.nyu.edu/cs/faculty/widlund/index.html). This author's research was supported in part by the U.S. Department of Energy under contracts DE-FG02-06ER25718 and DE-FC02-01ER25482 and in part by the National Science Foundation under grant NSF-DMS-0513251.
} 
note that the theory (and practice) of BDDC methods for conforming finite elements is by now quite well developed; see [23, 26, 25].

In a FETI-DP method, a linear system, formulated for a set of Lagrange multipliers, is solved after eliminating the displacement variables. The resulting linear system, in itself, contains a coarse problem, which also is directly related to the primal constraints discussed above, i.e., they are given by matching conditions on averages over edges/faces and/or by enforcing continuity of the solutions at vertices. Its preconditioner, on the other hand, is built only from subdomain problems, while for a BDDC method a linear system in the original degrees of freedom is solved in an iteration with a preconditioner that has both coarse and subdomain components. This appears to provide the BDDC methods with more flexibility, e.g., in allowing for the use of inexact coarse problems. Thus, for standard finite element problems an inexact coarse problem can be introduced by applying the BDDC method recursively to the coarse problem; see Tu [30, 29] and a recent conference paper by Mandel, Sousedík, and Dohrmann [27]. The use of inexact local problems for the BDDC preconditioners has also been considered by $\mathrm{Li}$ and Widlund [24]. We also note that Klawonn and Rheinbach [17] have developed and extensively tested algorithms which use inexact solvers for the coarse problem of FETI-DP methods.

There are a number of articles on solving the algebraic problems given by the mortar discretizations considered in this paper; see [32] and the literature cited therein. Most of them concern the simpler case of geometrically conforming partitioning of the original region $\Omega$; see, however, Achdou, Maday, and Widlund [1], where some iterative substructuring methods are developed and analyzed for problems in two dimensions in the geometrically nonconforming case, and Kim and Widlund [13], where an additive Schwarz method with overlap is designed and analyzed. Among the papers on the geometrically conforming case that are related to this paper, we mention $[14,12]$, where a Neumann-Dirichlet version of a FETI-DP method is analyzed. In [6], a FETI-DP method is considered, which is a generalization of a variant known for the standard conforming discretization. To the best of our knowledge, BDDC methods for the mortar discretization have not previously been discussed in the literature even for the geometrically conforming case.

A condition number bound of the form $C(1+\log (H / h))^{2}$ was first given for the BDDC operator by Mandel and Dohrmann [26] for a standard conforming discretization. This bound is of the same quality as the FETI-DP methods. In fact, the BDDC methods have been shown to be closely related to the FETI-DP methods. Thus, Mandel, Dohrmann, and Tezaur [25] have shown that the eigenvalues of the FETI-DP and BDDC operators are the same except possibly for eigenvalues equal to 0 and 1 . More recently, a new formulation of the BDDC method was given by Li and Widlund [23]. They introduced a change of variables as well as an average operator for the BDDC method closely related to the jump operator used in [19] in the analysis of FETI-DP methods. The change of variables greatly simplifies the analysis; it has also led to a successful and robust implementation of FETI-DP methods; see [16, 18]. We note that the idea of changing the variables for FETI-DP algorithms was discussed already in [20]. We also note that FETI-DP algorithms have also been implemented using enough point constraints to assure that there are no floating subdomains. In addition, optional admissible primal constraints (e.g., averages over edges or faces) are added to enhance the rate of convergence of the iterations; see [9]. These constraints are then handled by a separate set of Lagrange multipliers. We note that in our context, we often have no point constraints, and therefore this second approach cannot be used.

In this paper, we will describe a BDDC method for mortar discretizations, after 
a brief introduction to mortar methods. We will use a change of variables, as in [23] and Klawonn and Widlund [21], which is related to the primal constraints over edges/faces. We will consider quite general geometrically nonconforming partitions, i.e., we will not make any assumptions that the intersection of the boundaries of a pair of subdomains is a full face, edge, or a subdomain vertex.

We will work with mortar methods without any continuity constraints at subdomain vertices. Our results are valid for the traditional mortar methods as well as the dual basis mortar methods first introduced by Wohlmuth [31, 32]. We propose a preconditioner with a certain matrix of weights $D$ and obtain the condition number bound, $C \max _{i}\left\{\left(1+\log \left(H_{i} / h_{i}\right)\right)^{2}\right\}$, under some assumptions on the geometrically nonconforming subdomain partition in three dimensions. When the algorithm is applied to a geometrically conforming partition in three dimensions or a geometrically nonconforming partition in two dimensions, we obtain the same bound without any assumption on the partition. The subdomain partition can have interfaces that are narrow faces and our bounds can be established for such quite general cases. Section 4 is devoted to proving our condition number bound in terms of a bound of an average operator $E_{D}$ in an appropriate norm.

In section 5, we show that our BDDC preconditioner is closely connected to the Neumann-Dirichlet preconditioner for the FETI-DP methods given in [14, 12]. Connections are established between the average and jump operators, and the spectra of the BDDC and FETI-DP methods are then shown to be the same except possibly for an eigenvalue equal to 1 .

Results of numerical experiments are reported in the final section and show that the FETI-DP and BDDC methods perform well and very similarly when the same set of primal constraints is selected.

Throughout this paper, $C$ denotes a generic constant that depends neither on the mesh parameters nor on the coefficients of the elliptic problems.

We note that this paper originated from two projects developed separately by the first and second authors; the contribution of the third began with a suggestion that a theory could be developed for the geometrically nonconforming case using tools similar to those of [23].

\section{Finite element spaces and mortar matching constraints.}

2.1. A model problem and the mortar methods. We consider a model elliptic problem in a polygonal/polyhedral domain $\Omega \subset \mathbb{R}^{2}\left(\mathbb{R}^{3}\right)$ : find $u \in H_{0}^{1}(\Omega)$ such that

$$
\int_{\Omega} \rho(x) \nabla u(x) \cdot \nabla v(x) d x=\int_{\Omega} f(x) v(x) d x \quad \forall v \in H_{0}^{1}(\Omega),
$$

where $\rho(x) \geq \rho_{0}>0$ and $f(x) \in L^{2}(\Omega)$.

We partition $\Omega$ into disjoint polygonal/polyhedral subdomains

$$
\bar{\Omega}=\bigcup_{i=1}^{N} \bar{\Omega}_{i} .
$$

As previously noted, the partition can be geometrically nonconforming; see further discussion below. We assume that $\rho(x)=\rho_{i}, x \in \Omega_{i}$ for some positive constant $\rho_{i}$.

We denote by $X_{i}$ the $P_{1}$-conforming finite element space on a quasi-uniform triangulation of the subdomain $\Omega_{i}$. The finite element meshes typically do not align 
across subdomain interfaces. The trace space of $X_{i}$ on $\partial \Omega_{i}$ is denoted by $W_{i}$. We will use the product spaces

$$
X:=\prod_{i=1}^{N} X_{i}, \quad W:=\prod_{i=1}^{N} W_{i} .
$$

For functions in these spaces, we will impose mortar matching conditions across the interfaces using suitable spaces of Lagrange multipliers. Some of these matching conditions will be enforced throughout the iteration; they are directly related to the primal subspace.

In a geometrically nonconforming partition, the intersection of the boundaries of neighboring subdomains may be only part of an edge/face of a subdomain. We define the entire interface by

$$
\Gamma=\left(\bigcup_{i j} \partial \Omega_{i} \cap \partial \Omega_{j}\right) \backslash \partial \Omega .
$$

Among the subdomain edges/faces, we select nonmortar (slave) edges/faces $F_{l}$ such that

$$
\bigcup_{l} \bar{F}_{l}=\bar{\Gamma}, \quad F_{l} \cap F_{k}=\emptyset, \quad l \neq k
$$

see Figure 1 for an example of the selection of the nonmortar edges. For the case when $\rho(x)$ are very different across the interface, it is beneficial to select the part with smaller $\rho_{i}$ as the nonmortar; see Assumption 4.2.

Since the subdomain partition can be geometrically nonconforming, a single nonmortar edge/face $F_{l} \subset \partial \Omega_{i}$ may intersect the boundaries of several other subdomains $\Omega_{j}$. This provides $F_{l}$ with a partition

$$
\bar{F}_{l}=\bigcup_{j} \bar{F}_{i j}, \quad F_{i j}=\partial \Omega_{i} \cap \partial \Omega_{j}
$$

see Figure 1 for the mortar counter parts of the nonmortar edge $F_{l}$. A dual or standard Lagrange multiplier space $M\left(F_{l}\right)$ is introduced for each nonmortar edge/face $F_{l}$. We require $M\left(F_{l}\right)$ to have the same dimension as the space

$$
\stackrel{\circ}{W}\left(F_{l}\right):=\left.W_{i}\right|_{F_{l}} \cap H_{0}^{1}\left(F_{l}\right),
$$

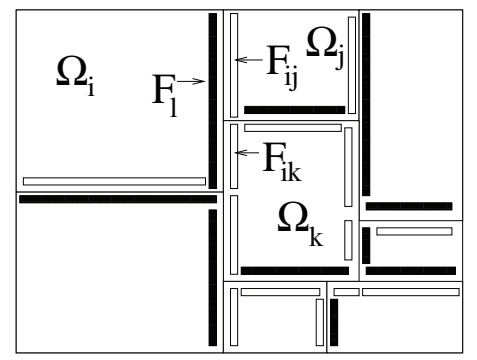

FIG. 1. Nonmortar edges (black) and mortar edges (white) in a geometrically nonconforming partition.

Copyright $\odot$ by SIAM. Unauthorized reproduction of this article is prohibited. 
that it is nonempty, and that it contains the constants. Constructions of such Lagrange multiplier spaces are given in $[2,3]$ using standard Lagrange multiplier spaces, and in [31,32] using dual Lagrange multiplier spaces; see also [11].

For $\left(w_{1}, \ldots, w_{N}\right) \in W, w_{i} \in W_{i}$, we define $\phi_{l} \in L^{2}\left(F_{l}\right)$ by $\phi_{l}=w_{j}$ on $F_{i j} \subset$ $F_{l}$. The mortar matching condition for the geometrically nonconforming partition is given by

$$
\int_{F_{l}}\left(w_{i}-\phi_{l}\right) \lambda d s=0 \quad \forall \lambda \in M\left(F_{l}\right), \forall F_{l} .
$$

The mortar finite element method for problem (2.1) amounts to approximating the solution of the continuous problem by a Galerkin method using the mortar finite element space

$$
\widehat{X}:=\left\{v \in X:\left.v\right|_{\Gamma} \text { satisfies the mortar matching condition }(2.3)\right\},
$$

where $\left.v\right|_{\Gamma}$ is the restriction of $v$ to the interface $\Gamma$. We introduce the space $\widehat{W}$ as the restriction of $\widehat{X}$ to $\Gamma$,

$$
\widehat{W}:=\left\{w: w=\left.v\right|_{\Gamma} \forall v \text { in } \widehat{X}\right\} .
$$

2.2. Finite element spaces and a change of variables. In this subsection, we introduce a change of variables for some of the unknowns in the space $W$. It is based on the primal constraints that will be specified for our BDDC method. In mortar discretizations, we may consider the following sets of primal constraints: vertex constraints; vertex and edge average constraints, or edge average constraints only, for two dimensions; and vertex constraints and face average constraints, or face average constraints only, for three dimensions. We note that vertex constraints are appropriate only for the first generation of the mortar methods, in which case the subdomain vertex values are constrained to be continuous. In order to reduce the number of primal constraints, we can also select only some edges/faces as primal. Such choices have been considered for the FETI-DP methods and conforming finite elements in [21], and for mortar finite elements in [15].

In our BDDC formulation, we will select primal constraints over edges/faces from the set of mortar matching constraints $(2.3)$. We consider $\left\{\lambda_{i j, k}\right\}_{k}$, the basis functions of $M\left(F_{l}\right)$ that are supported in $\bar{F}_{i j} \subset \bar{F}_{l}$, and define

$$
\lambda_{i j}=\sum_{k} \lambda_{i j, k}
$$

We assume that at least one such basis function $\lambda_{i j, k}$ exists for each $F_{i j}$.

We now introduce one primal constraint over each interface $F_{i j} \subset F_{l}$ and for all edges/faces $F_{l}$,

$$
\int_{F_{i j}}\left(w_{i}-w_{j}\right) \lambda_{i j} d s=0
$$

and define

$$
\widetilde{W}=\{w \in W: w \text { satisfies the primal constraints }(2.5)\}
$$

Copyright (C) by SIAM. Unauthorized reproduction of this article is prohibited. 
We note that $\widehat{W} \subset \widetilde{W} \subset W$, where $\widehat{W}$ is the restriction of $\widehat{X}$ to $\Gamma$. For the case of a geometrically conforming partition, i.e., when each $F_{i j}$ is a full edge/face of two subdomains, these constraints are edge/face average matching conditions because $\lambda_{i j}=1$. In addition to these constraints, vertex constraints can be considered but only if the partition is geometrically conforming.

Throughout this paper, we use hats for functions and function spaces that satisfy all of the mortar matching conditions. We use tildes for functions and function spaces that satisfy only the primal constraints across the subdomain interface.

Following Li and Widlund [23], we now introduce a change of variables based on the primal constraints. We provide details for the two-dimensional case but note that this approach can be extended to the three-dimensional case without any difficulty.

We recall that $F_{l} \subset \partial \Omega_{i}$, denoted from now on by $F$, is a nonmortar edge/face and that $\left\{F_{i j}\right\}_{j}$ is a partition of $F$ given by $F_{i j}=F \cap \partial \Omega_{j}$, a mortar edge/face of $\Omega_{j}$. We denote by $\left\{v_{k}\right\}_{k=1}^{L}$ the values of the unknowns of $w_{i} \in W_{i}$ at the nodes on $F_{i j}$, with nodal basis functions that are supported in $\bar{F}_{i j}$, and by $\left\{\eta_{k}\right\}_{k=1}^{p}$ the other unknowns on $\bar{F}_{i j}$. We will now define a transformation that retains the unknowns $\left\{\eta_{k}\right\}_{k=1}^{p}$ and changes $\left\{v_{k}\right\}_{k=1}^{L}$ into $\left\{\xi_{k}\right\}_{k=1}^{L}$ as follows: we pick one unknown $\xi_{m}$ among $\left\{\xi_{k}\right\}_{k=1}^{L}$ and build a transformation $T_{F_{i j}}$ so that

$$
\left(\begin{array}{l}
\eta \\
v
\end{array}\right)=T_{F_{i j}}\left(\begin{array}{l}
\eta \\
\xi
\end{array}\right), \quad \xi_{m}=\frac{\int_{F_{i j}} w_{i} \lambda_{i j} d s}{\int_{F_{i j}} \lambda_{i j} d s} .
$$

Here $\eta, v$, and $\xi$ denote vectors of the unknowns $\left\{\eta_{k}\right\}_{k=1}^{p},\left\{v_{k}\right\}_{k=1}^{L}$, and $\left\{\xi_{k}\right\}_{k=1}^{L}$, respectively.

Let

$$
A_{\eta_{k}}=\frac{\int_{F_{i j}} \phi_{\eta_{k}} \lambda_{i j} d s}{\int_{F_{i j}} \lambda_{i j} d s}, \quad A_{v_{k}}=\frac{\int_{F_{i j}} \phi_{v_{k}} \lambda_{i j} d s}{\int_{F_{i j}} \lambda_{i j} d s}
$$

where $\phi_{\eta_{k}}$ and $\phi_{v_{k}}$ are the nodal basis functions of the unknowns $\eta_{k}$ and $v_{k}$, respectively. To make the presentation simpler, we assume that $p=2$, but what follows can be generalized to any $p$. We will use the following transformation $T_{F_{i j}}$ :

$$
\left(\begin{array}{c}
\eta_{1} \\
\eta_{2} \\
v_{1} \\
\vdots \\
v_{m-1} \\
v_{m} \\
v_{m+1} \\
\vdots \\
v_{L}
\end{array}\right)=T_{F_{i j}}\left(\begin{array}{c}
\eta_{1} \\
\eta_{2} \\
\xi_{1} \\
\vdots \\
\xi_{m-1} \\
\xi_{m} \\
\xi_{m+1} \\
\vdots \\
\xi_{L}
\end{array}\right)=\left(\begin{array}{ccccccccc}
1 & 0 & 0 & \cdots & 0 & 0 & 0 & \cdots & 0 \\
0 & 1 & 0 & \cdots & 0 & 0 & 0 & \cdots & 0 \\
0 & 0 & 1 & \cdots & 0 & A & 0 & \cdots & 0 \\
\vdots & \vdots & \vdots & \ddots & \vdots & \vdots & \vdots & \ddots & \vdots \\
0 & 0 & 0 & \cdots & 1 & A & 0 & \cdots & 0 \\
c_{1} & c_{2} & r_{1} & \cdots & r_{m-1} & A & r_{m+1} & \cdots & r_{L} \\
0 & 0 & 0 & \cdots & 0 & A & 1 & \cdots & 0 \\
\vdots & \vdots & \vdots & \ddots & \vdots & \vdots & \vdots & \ddots & \vdots \\
0 & 0 & 0 & \cdots & 0 & A & 0 & \cdots & 1
\end{array}\right)
$$




$$
\left(\begin{array}{c}
\eta_{1} \\
\eta_{2} \\
\xi_{1} \\
\vdots \\
\xi_{m-1} \\
\xi_{m} \\
\xi_{m+1} \\
\vdots \\
\xi_{L}
\end{array}\right)=A\left(\begin{array}{c}
0 \\
0 \\
1 \\
\vdots \\
\vdots \\
\vdots \\
\vdots \\
\vdots \\
\vdots \\
\vdots \\
1
\end{array}\right) \xi_{m}+\left(\begin{array}{c}
\eta_{1} \\
\eta_{2} \\
\xi_{1} \\
\vdots \\
\xi_{m-1} \\
\xi_{0} \\
\xi_{m+1} \\
\vdots \\
\vdots \\
\vdots \\
\xi_{L}
\end{array}\right)
$$

where

$$
\xi_{0}=c_{1} \eta_{1}+c_{2} \eta_{2}+r_{1} \xi_{1}+\cdots+r_{m-1} \xi_{m-1}+r_{m+1} \xi_{m+1}+\cdots+r_{L} \xi_{L}
$$

and

$$
A=\frac{\int_{F_{i j}} \lambda_{i j} d s}{\sum_{k=1}^{L} A_{v_{k}}}, \quad c_{1}=-\frac{A_{\eta_{1}}}{A_{v_{m}}}, \quad c_{2}=-\frac{A_{\eta_{2}}}{A_{v_{m}}}, \quad r_{k}=-\frac{A_{v_{k}}}{A_{v_{m}}}, \quad k \neq m .
$$

We can then see that this transformation satisfies the (2.7) requirement. The transformation $T_{F_{i j}}$ can be applied to each face $F_{i j} \subset F$ independently, since it does not change any nodal values other than $\left\{v_{k}\right\}_{k=1}^{L}$, which are associated with the unknowns of the nodes interior to $F_{i j}$.

On the other side, the mortar side, of the interface $F_{i j}$, i.e., $F_{i j} \subset \partial \Omega_{j}$, we perform a change of basis to the unknowns in finite element space $W_{j}$. In this case, we introduce another set of unknowns $\left\{v_{k}\right\}_{k=1}^{J}$ and $\left\{\eta_{k}\right\}_{k=1}^{p}$. The unknowns $\left\{v_{k}\right\}_{k=1}^{J}$ are related to the nodes on $F_{i j}$ with nodal basis functions, which belong to $W_{j}$ and are supported in $\bar{F}_{i j}$. The unknowns $\left\{\eta_{k}\right\}_{k=1}^{p}$ are the remaining unknowns on $F_{i j}$. The transformation $T_{F_{i j}}$ is then defined for these unknowns similarly as for a nonmortar interface.

Using the transforms $T_{F_{i j}}$, we represent the Schur complement of the local stiffness and the mortar matching matrices, and the local force vector in the space of the new unknowns by

$$
T^{(i)^{t}} S^{(i)} T^{(i)}, \quad B^{(i)} T^{(i)}, \quad T^{(i)^{t}} g^{(i)}
$$

Here $S^{(i)}$ is the reduced matrix obtained after eliminating all variables associated with only the subdomain $\Omega_{i}$, and $T^{(i)}$ designates the transform of the original unknowns into the new unknowns of the subdomain boundary $\partial \Omega_{i}$. In the following, we will use the same notation, $S^{(i)}, B^{(i)}$, and $g^{(i)}$, for the matrices and vectors obtained after the change of unknowns, to simplify the notation. We will also use the notation $W_{i}$ for the space of the new unknowns.

The unknowns $\xi_{m}$ in (2.7), representing certain weighted averages over the edges, are the primal variables. Using the new variables, the space $\widetilde{W}$, defined in (2.6), can be represented as

$$
\widetilde{W}=W_{\Delta} \oplus \widehat{W}_{\Pi},
$$


where $W_{\Delta}$ consists of the vectors of unknowns which are not primal unknowns, and $\widehat{W}_{\Pi}$ consists of the vectors of global, primal unknowns.

We now derive the matrix representation of the mortar matching condition (2.3) in the space $\widetilde{W}$ of the new unknowns. The mortar matching condition (2.3) is redundant when enforced for the functions in the space $\widetilde{W}$. We recall that $\left\{\lambda_{i j, k}\right\}_{k}$ are the Lagrange multiplier basis elements supported in $F_{i j}$. To make the mortar matching condition nonredundant, we eliminate one basis element among $\left\{\lambda_{i j, k}\right\}_{k}$ for each $F_{i j} \subset$ $F_{l}$, and we denote the reduced Lagrange multiplier space by $\frac{}{M}\left(F_{l}\right)$. The entire nonredundant Lagrange multiplier space is then defined as

$$
\bar{M}=\prod_{l} \bar{M}\left(F_{l}\right) .
$$

The remaining nonprimal, mortar matching conditions of (2.3) are enforced using the reduced space $\bar{M}\left(F_{l}\right)$. In matrix form, this can be written as

$$
B_{\Delta} w_{\Delta}+B_{\Pi} w_{\Pi}=0 .
$$

The space $W_{\Delta}$ can be split into

$$
W_{\Delta}=W_{\Delta, n} \oplus W_{\Delta, m},
$$

where $n$ and $m$ denote unknowns in the interior of the nonmortar edges/faces and the remaining unknowns, respectively. The mortar matching conditions can then be written as

$$
B_{n} w_{n}+B_{m} w_{m}+B_{\Pi} w_{\Pi}=0 .
$$

Since these equations are obtained using only the nonredundant Lagrange multiplier space $\bar{M}$, the matrix $B_{n}$ is invertible.

After a symmetric permutation, we can write the local Schur complement and the local Schur complement vector as

$$
S^{(i)}=\left(\begin{array}{cc}
S_{\Delta \Delta}^{(i)} & S_{\Delta \Pi}^{(i)} \\
S_{\Pi \Delta}^{(i)} & S_{\Pi \Pi}^{(i)}
\end{array}\right), \quad g^{(i)}=\left(\begin{array}{c}
g_{\Delta}^{(i)} \\
g_{\Pi}^{(i)}
\end{array}\right),
$$

and define a partially subassembled matrix and two vectors by

$$
\widetilde{S}=\left(\begin{array}{cc}
S_{\Delta \Delta} & S_{\Delta \Pi} \\
S_{\Pi \Delta} & S_{\Pi \Pi}
\end{array}\right), \quad g_{\Delta}=\left(\begin{array}{c}
g_{\Delta}^{(1)} \\
\vdots \\
g_{\Delta}^{(N)}
\end{array}\right), \quad g_{\Pi}=\sum_{i=1}^{N} R_{\Pi}^{(i)^{t}} g_{\Pi}^{(i)},
$$

where

$$
\begin{aligned}
S_{\Delta \Delta} & =\operatorname{diag}_{i=1}^{N}\left(S_{\Delta \Delta}^{(i)}\right), \\
S_{\Pi \Delta} & =\left(\begin{array}{lll}
R_{\Pi}^{(1)^{t}} S_{\Pi \Delta}^{(1)} & \cdots & R_{\Pi}^{(N)^{t}} S_{\Pi \Delta}^{(N)}
\end{array}\right), \quad S_{\Delta \Pi}=S_{\Pi \Delta}^{t}, \\
S_{\Pi \Pi} & =\sum_{i=1}^{N} R_{\Pi}^{(i)}{ }^{t} S_{\Pi \Pi}^{(i)} R_{\Pi}^{(i)} .
\end{aligned}
$$

Here $R_{\Pi}^{(i)}$ is the restriction of the global primal unknowns to the subdomain primal unknowns. The matrix $\widetilde{S}$ is central to the description of our BDDC algorithm. 
3. A BDDC method for the mortar discretizations. In this section, we will define a BDDC operator for the discrete elliptic problem described in section 2.1. We consider the same finite element space and subdomain partition as in section 2.1 and, as in section 2.2, we will work with the unknowns obtained after the change of variables.

Since the matrix $B_{n}$ of (2.10) is invertible, we can solve for $w_{n}$,

$$
w_{n}=-B_{n}^{-1}\left(B_{m} w_{m}+B_{\Pi} w_{\Pi}\right) .
$$

We next define the matrix

$$
R_{\Gamma}=\left(\begin{array}{cc}
-B_{n}^{-1} B_{m} & -B_{n}^{-1} B_{\Pi} \\
I & 0 \\
0 & I
\end{array}\right),
$$

which maps $\left(w_{m}^{t}, w_{\Pi}^{t}\right)^{t}$ into a vector $\left(w_{n}^{t}, w_{m}^{t}, w_{\Pi}^{t}\right)^{t}$ that satisfies the mortar matching condition (2.10). The mortar finite element space of section 2.1 can then be characterized as

$$
\widehat{W}=\left\{w \in \widetilde{W}:\left(w_{n}, w_{m}, w_{\Pi}\right) \text { satisfies }(2.10)\right\} .
$$

In the BDDC method, we work with the following discrete problem:

$$
R_{\Gamma}^{t} \widetilde{S} R_{\Gamma}\left(\begin{array}{c}
w_{m} \\
w_{\Pi}
\end{array}\right)=R_{\Gamma}^{t}\left(\begin{array}{c}
g_{m} \\
g_{\Pi}
\end{array}\right)
$$

where $g_{m}$ is the component of the vector $g_{\Delta}$ in (2.11) not related to the nonmortar part.

Let us now define, with $R_{\Gamma}$ given by (3.1),

$$
R_{D, \Gamma}=D R_{\Gamma}=\left(\begin{array}{ccc}
D_{n n} & & \\
& D_{m m} & \\
& & D_{\Pi \Pi}
\end{array}\right) R_{\Gamma},
$$

where the scaling matrices are selected to be

$$
D_{n n}=0, \quad D_{m m}=I, \quad D_{\Pi \Pi}=I .
$$

We now propose the following preconditioner:

$$
M^{-1}=R_{D, \Gamma}^{t} \widetilde{S}^{-1} R_{D, \Gamma}
$$

for problem (3.2). Using the block Cholesky decomposition of $\widetilde{S}$ as in Li and Widlund [23], we have

$$
\widetilde{S}^{-1}=\left(\begin{array}{cc}
S_{\Delta \Delta}^{-1} & 0 \\
0 & 0
\end{array}\right)+\Psi^{t} F_{\Pi \Pi}^{-1} \Psi
$$

where

$$
\begin{aligned}
F_{\Pi \Pi} & =\sum_{i=1}^{N}\left(R_{\Pi}^{(i)}\right)^{t}\left(S_{\Pi \Pi}^{(i)}-S_{\Pi \Delta}^{(i)} S_{\Delta \Delta}^{(i)}{ }^{-1} S_{\Delta \Pi}^{(i)}\right) R_{\Pi}^{(i)}, \\
\Psi^{t} & =R_{\Pi}^{t}-\sum_{i=1}^{N}\left(R_{\Delta}^{(i)}\right)^{t}\left(S_{\Delta \Delta}^{(i)}\right)^{-1} S_{\Delta \Pi}^{(i)} R_{\Pi}^{(i)} .
\end{aligned}
$$

Copyright (c) by SIAM. Unauthorized reproduction of this article is prohibited. 
Here $R_{\Pi}^{(i)}: \widehat{W}_{\Pi} \rightarrow W_{\Pi}^{(i)}$ is the restriction of the global primal variables to those of the subdomain $\Omega_{i}$, and $R_{\Pi}^{t}: \widehat{W}_{\Pi} \rightarrow W_{\Delta} \oplus \widehat{W}_{\Pi}$ and $\left(R_{\Delta}^{(i)}\right)^{t}: W_{\Delta}^{(i)} \rightarrow W_{\Delta} \oplus \widehat{W}_{\Pi}$ provide extensions by zero. The columns of the matrix $\Psi$ are coarse basis functions of minimal energy with the value 1 at one of the primal unknowns and vanishing at the other primal unknowns; see [5].

The BDDC operator of the problem, given in (3.2), with the preconditioner $M^{-1}$, given in (3.5), is then given by

$$
B_{D D C}=R_{D, \Gamma}^{t} \widetilde{S}^{-1} R_{D, \Gamma} R_{\Gamma}^{t} \widetilde{S} R_{\Gamma} .
$$

4. Condition number analysis using a bound on $\boldsymbol{E}_{D}$. In this section, we will estimate the condition number of the BDDC operator by using the approach introduced in [22]. A bound for the average operator $E_{D}$ in the $\widetilde{S}$-norm is central in the analysis; see below. For definitions of $R_{\Gamma}$ and $R_{D, \Gamma}$, see (3.1) and (3.3), respectively. The operator $E_{D}$ is defined by

$$
E_{D}=R_{\Gamma} R_{D, \Gamma}^{t}
$$

In the following, we will show that the weight matrix $D$ has been chosen so that

$$
\begin{gathered}
R_{\Gamma}^{t} R_{D, \Gamma}=R_{D, \Gamma}^{t} R_{\Gamma}=I \\
\left|E_{D} w\right|_{\widetilde{S}}^{2} \leq C \max _{i}\left\{\left(1+\log \frac{H_{i}}{h_{i}}\right)^{2}\right\}|w|_{\widetilde{S}}^{2} .
\end{gathered}
$$

Here $|w|_{\widetilde{S}}^{2}=\langle\widetilde{S} w, w\rangle$. We then consider

$$
R_{\Gamma}^{t} R_{D, \Gamma}\left(\begin{array}{c}
w_{m} \\
w_{\Pi}
\end{array}\right)=\left(\begin{array}{c}
-B_{m}^{t}\left(B_{n}^{t}\right)^{-1} D_{n n} z_{n}+D_{m m} w_{m} \\
-B_{\Pi}^{t}\left(B_{n}^{t}\right)^{-1} D_{n n} z_{n}+D_{\Pi \Pi} w_{\Pi}
\end{array}\right)
$$

where

$$
z_{n}=-B_{n}^{-1}\left(B_{m} w_{m}+B_{\Pi} w_{\Pi}\right)
$$

We recall the scaling factors of the weight matrix $D$ in (3.4) and we can easily see that these weights give the (P1) property.

Remark 4.1. The weights above lead to an operator $E_{D}$ of the form

$$
E_{D}\left(\begin{array}{l}
w_{n} \\
w_{m} \\
w_{\Pi}
\end{array}\right)=\left(\begin{array}{c}
-B_{n}^{-1}\left(B_{m} w_{m}+B_{\Pi} w_{\Pi}\right) \\
w_{m} \\
w_{\Pi}
\end{array}\right) .
$$

In contrast to the case of conforming finite elements, this does not involve any averaging across the interface. We will still call $E_{D}$ the average operator, borrowing the name from the conforming case.

We will now show that the average operator $E_{D}$ satisfies the (P2) property for the weight matrix $D$ just given. As a preparation, we need to establish an estimate for the mortar projection of a function $w$ in $\widetilde{W}$ in the $H_{00}^{1 / 2}(F)$-norm. For an edge/face $F \subset \partial \Omega_{i}$, the space $H_{00}^{1 / 2}(F)$ consists of the functions for which the zero extension to the whole boundary $\partial \Omega_{i}$ belongs to the Sobolev space $H^{1 / 2}\left(\partial \Omega_{i}\right)$. It is equipped with the norm

$$
\|w\|_{H_{00}^{1 / 2}(F)}^{2}=|w|_{H^{1 / 2}(F)}^{2}+\int_{F} \frac{|w(x)|^{2}}{\operatorname{dist}(x, \partial F)} d s(x) .
$$


This norm has the well-known property that

$$
c|\widetilde{w}|_{H^{1 / 2}\left(\partial \Omega_{i}\right)} \leq\|w\|_{H_{00}^{1 / 2}(F)} \leq C|\widetilde{w}|_{H^{1 / 2}\left(\partial \Omega_{i}\right)},
$$

where $\widetilde{w}$ is the zero extension of $w$ to $\partial \Omega_{i} \backslash F$; see [10, Lemma 1.3.2.6].

We recall that a nonmortar edge/face $F$ of $\partial \Omega_{i}$ is a union of mortar interfaces $F_{i j}$ common to $\partial \Omega_{i}$ and $\partial \Omega_{j}$. We recall that $\phi$ is a function defined on $F$ with $\phi=w_{j}$ on each $F_{i j} \subset F$, and with $w_{j} \in W_{j}$, the finite element space provided for $\partial \Omega_{j}$. We then have $\phi \in H^{1 / 2-\epsilon}(F)$ for any $\epsilon>0$. Because of the slightly weaker regularity of the function $\phi$, caused by the geometrically nonconforming partition, we have some difficulty obtaining the condition number bound with only two logarithmic factors for geometrically nonconforming partitions in three dimensions. We will overcome this difficulty by using an additional finite element space for the interface $F_{i j}$ and an $L^{2}$-projection onto this space. This will result in a condition number bound with two logarithmic factors under some assumptions on the geometry of the subdomain partition; see Assumption 4.3 below.

We also need the following assumption on the coefficients of the elliptic problem. We note that this assumption basically reflects a weakness of the mortar methods in the case of geometrically nonconforming partitions.

Assumption 4.2. The coefficients satisfy

$$
\rho_{i} \leq C \rho_{j},
$$

where $\Omega_{i}$ and $\Omega_{j}$ correspond to the nonmortar and mortar side of the common set $F_{i j}=\partial \Omega_{i} \cap \partial \Omega_{j}$, respectively.

We also will use the following assumption.

ASSUMPTION 4.3. A geometrically nonconforming partition $\left\{\Omega_{i}\right\}_{i}$ in three dimensions satisfies the following three assumptions.

1. The subdomains are polytopes.

2. A quasi-uniform triangulation, with a mesh size comparable to $h_{i}$, is possible for the interface $F_{i j}$.

3. Any subdomain has a diameter comparable to those of its neighbors.

We recall that the finite element space $\stackrel{\circ}{W}(F)$, given in $(2.2)$, and a Lagrange multiplier space $M(F)$ are provided for the nonmortar edge/face $F$. We now define the mortar projection.

DEFINITION 4.4. The mortar projection $\pi_{F}: L^{2}(F) \rightarrow \stackrel{\circ}{W}(F)$ of the nonmortar edge/face $F$ is defined by

$$
\int_{F}\left(v-\pi_{F}(v)\right) \lambda d s=0 \quad \forall \lambda \in M(F) .
$$

This mortar projection has been shown to be stable in the $L^{2}$ - and $H_{00}^{1 / 2}$-norms in $[3,2,32]$.

LEMma 4.5. Under Assumptions 4.2 and 4.3 and with $w=\left(w_{1}, \ldots, w_{N}\right) \in \widetilde{W}$, we have

$$
\rho_{i}\left\|\pi_{F}\left(\phi-w_{i}\right)\right\|_{H_{00}^{1 / 2}(F)}^{2} \leq C\left(1+\log \frac{H_{i}}{h_{i}}\right)^{2} \sum_{k \in I(F)}\left\langle S^{(k)} w_{k}, w_{k}\right\rangle .
$$

Here $F \subset \partial \Omega_{i}$ is an edge/face, $\phi=w_{j}$ on $F_{i j} \subset F$, and $I(F)$ is the set of indices of the subdomains with boundaries that intersect $F$.

Copyright $@$ by SIAM. Unauthorized reproduction of this article is prohibited. 
Proof. We will prove the result for a geometrically nonconforming partition in three dimensions under Assumption 4.3. In the case of a geometrically conforming partition in three dimensions and for any partition in two dimensions, the same result can be obtained straightforwardly without any assumption on the partition.

For each interface $F_{i j}$, we define a characteristic function $\chi_{i j} \in L^{2}(F)$ with the value 1 on $F_{i j}$ and the value 0 on $F \backslash F_{i j}$. In addition, we introduce a quasi-uniform finite element space $U\left(F_{i j}\right)$ on the interface $F_{i j}$ with a mesh size comparable to $h_{i}$, that of the finite element space $W_{i}$ of the subdomain $\Omega_{i}$ of the nonmortar side. The $L^{2}$-projection onto $U\left(F_{i j}\right)$ is denoted by $Q_{i j}$ and it satisfies the following properties (see [4, Chapter II]: $\forall w \in H^{1 / 2}\left(F_{i j}\right)$ ):

$$
\left\|w-Q_{i j} w\right\|_{L^{2}\left(F_{i j}\right)}^{2} \leq C h_{i}|w|_{H^{1 / 2}\left(F_{i j}\right)}^{2}, \quad\left\|Q_{i j} w\right\|_{H^{1 / 2}\left(F_{i j}\right)}^{2} \leq C\|w\|_{H^{1 / 2}\left(F_{i j}\right)}^{2},
$$

where the $L^{2}$-term in the $H^{1 / 2}$-norm is scaled by $1 /\left|F_{i j}\right|$. Here $\left|F_{i j}\right|$ is the diameter of $F_{i j}$.

Then, on $F$, consider

$$
\begin{aligned}
w_{i}-\phi & =\sum_{j} \chi_{i j}\left(w_{i}-w_{j}\right) \\
& =\sum_{j} \chi_{i j}\left(\left(w_{i}-c_{i j}\right)-\left(w_{j}-c_{i j}\right)\right) .
\end{aligned}
$$

Here $c_{i j}$ denotes the common average value of $w_{i}$ and $w_{j}$ defined by

$$
c_{i j}=\frac{\int_{F_{i j}} w_{i} \lambda_{i j} d s}{\int_{F_{i j}} \lambda_{i j} d s}=\frac{\int_{F_{i j}} w_{j} \lambda_{i j} d s}{\int_{F_{i j}} \lambda_{i j} d s},
$$

where $\lambda_{i j}$ are defined in $(2.4) ; c_{i j}$ is closely related to the primal mortar matching condition (2.5).

It suffices to show that

$$
\left\|\pi_{F}\left(\chi_{i j}\left(w_{j}-c_{i j}\right)\right)\right\|_{H_{00}^{1 / 2}(F)}^{2} \leq C\left(1+\log \frac{H_{i}}{h_{i}}\right)^{2}\left|w_{j}\right|_{H^{1 / 2}\left(\partial \Omega_{j}\right)}^{2},
$$

and to give a similar estimate for $w_{i}-c_{i j}$. We will prove (4.4) but leave out the estimate for $w_{i}-c_{i j}$, which is quite similar. The required estimate then follows from Assumption 4.2 and the fact that $\left|w_{j}\right|_{H^{1 / 2}\left(\partial \Omega_{j}\right)}^{2}$ is spectrally equivalent to $\left(1 / \rho_{j}\right)\left\langle S^{(j)} w_{j}, w_{j}\right\rangle$.

Let

$$
z=w_{j}-c_{i j}
$$

We decompose $Q_{i j}(z)$ into

$$
Q_{i j}(z)=I_{F_{i j}}\left(Q_{i j}(z)\right)+I_{\partial F_{i j}}\left(Q_{i j}(z)\right),
$$

where the first term is equal to $Q_{i j}(z)$ at all interior nodal points of $F_{i j}$ and vanishes on $\partial F_{i j}$ while the second term is equal to $Q_{i j}(z)$ at the nodal points of $\partial F_{i j}$ and vanishes at the remaining nodal points of $F_{i j}$. We have

$$
\begin{aligned}
\left\|\pi_{F}\left(\chi_{i j}\left(w_{j}-c_{i j}\right)\right)\right\|_{H_{00}^{1 / 2}(F)}^{2} & =\left\|\pi_{F}\left(\chi_{i j} z\right)\right\|_{H_{00}^{1 / 2}(F)}^{2} \\
& \leq 2\left\|\pi_{F}\left(\chi_{i j}\left(z-Q_{i j}(z)\right)\right)\right\|_{H_{00}^{1 / 2}(F)}^{2} \\
& +2\left\|\pi_{F}\left(\chi_{i j} Q_{i j}(z)\right)\right\|_{H_{00}^{1 / 2}(F)}^{2} .
\end{aligned}
$$

Copyright $@$ by SIAM. Unauthorized reproduction of this article is prohibited. 
The first term above is estimated by

$$
\begin{aligned}
\left\|\pi_{F}\left(\chi_{i j}\left(z-Q_{i j}(z)\right)\right)\right\|_{H_{00}^{1 / 2}(F)}^{2} & \leq C h_{i}^{-1}\left\|\chi_{i j}\left(z-Q_{i j}(z)\right)\right\|_{L^{2}(F)}^{2} \\
& =C h_{i}^{-1}\left\|z-Q_{i j}(z)\right\|_{L^{2}\left(F_{i j}\right)}^{2} \\
& \leq C|z|_{H^{1 / 2}\left(F_{i j}\right)}^{2} \\
& \leq C\left|w_{j}\right|_{H^{1 / 2}\left(\partial \Omega_{j}\right)} .
\end{aligned}
$$

We have used an inverse inequality, the $L^{2}$-stability of $\pi_{F}$, and the properties of $Q_{i j}(z)$ given in (4.3).

There remains for us to estimate the second term of (4.6). By Assumption 4.3, the subdomain interfaces $F_{i j}$ are polygonal regions. For a geometrically nonconforming partition, the area of the interface $F_{i j}$ might be comparable to that of $F_{j}$, the face of $\Omega_{j}$ such that $F_{j} \cap \partial \Omega_{i}=F_{i j}$. In the other case, when $F_{i j}$ is only a small part of $F_{j}$, it could be a narrow strip, e.g., $[0, H] \times[0, \delta]$, or a rectangular region with its area comparable to $[0, \delta] \times[0, \delta]$, where $\delta$ is comparable to the mesh size $h$.

We will first consider the second term in (4.6) when the area of the interface $F_{i j}$ is comparable to that of $F_{j}$. Using (4.5), we have

$$
\begin{aligned}
\left\|\pi_{F}\left(\chi_{i j} Q_{i j}(z)\right)\right\|_{H_{00}^{1 / 2}(F)}^{2} & =\left\|\pi_{F}\left(\chi_{i j}\left(I_{F_{i j}} Q_{i j}(z)+I_{\partial F_{i j}} Q_{i j}(z)\right)\right)\right\|_{H_{00}^{1 / 2}(F)}^{2} \\
& \leq C\left(\left\|\widetilde{I}_{F_{i j}}\left(Q_{i j}(z)\right)\right\|_{H_{00}^{1 / 2}(F)}^{2}+h_{i}^{-1}\left\|\widetilde{I}_{\partial F_{i j}} Q_{i j}(z)\right\|_{L^{2}(F)}^{2}\right) \\
& \leq C\left(\left\|I_{F_{i j}}\left(Q_{i j}(z)\right)\right\|_{H_{00}^{1 / 2}\left(F_{i j}\right)}^{2}+\left\|I_{\partial F_{i j}} Q_{i j}(z)\right\|_{L^{2}\left(\partial F_{i j}\right)}^{2}\right),
\end{aligned}
$$

where $\widetilde{I}_{F_{i j}}(v)$ and $\widetilde{I}_{\partial F_{i j}}(v)$ are the extensions of $I_{F_{i j}}(v)$ and $I_{\partial F_{i j}}(v)$ by zero, respectively. Here, we have used an inverse inequality, the stability of $\pi_{F}$ in the $L^{2}$ - and $H_{00}^{1 / 2}$-norms, and the following inequalities:

$$
\begin{gathered}
\left\|\widetilde{I}_{F_{i j}}\left(Q_{i j}(z)\right)\right\|_{H_{00}^{1 / 2}(F)} \leq\left\|I_{F_{i j}}\left(Q_{i j}(z)\right)\right\|_{H_{00}^{1 / 2}\left(F_{i j}\right)}, \\
\left\|\widetilde{I}_{\partial F_{i j}} Q_{i j}(z)\right\|_{L^{2}(F)}^{2} \leq C h_{i}\left\|I_{\partial F_{i j}} Q_{i j}(z)\right\|_{L^{2}\left(\partial F_{i j}\right)}^{2} .
\end{gathered}
$$

By applying Lemmas $4.17,4.19$, and 4.24 of [28] to the terms of (4.8), and using (4.3) and the Poincaré inequality, we obtain

$$
\left\|\pi_{F}\left(\chi_{i j} Q_{i j}(z)\right)\right\|_{H_{00}^{1 / 2}(F)}^{2} \leq C\left(1+\log \frac{H_{i j}}{h_{i}}\right)^{2}\left|w_{j}\right|_{H^{1 / 2}\left(\partial \Omega_{j}\right)}^{2}
$$

where $H_{i j}$ is the diameter of $F_{i j}$, which satisfies $H_{i j} \leq H_{i}$.

We now consider the second term in (4.6) for the case when $F_{i j}$ is only a small part of $F_{j}$. Then,

$$
\begin{aligned}
\left\|\pi_{F}\left(\chi_{i j} Q_{i j}(z)\right)\right\|_{H_{00}^{1 / 2}(F)}^{2} & \leq C h_{i}^{-1}\left\|\pi_{F}\left(\chi_{i j} Q_{i j}(z)\right)\right\|_{L^{2}(F)}^{2} \\
& \leq C h_{i}^{-1}\|z\|_{L^{2}\left(F_{i j}\right)}^{2}=C h_{i}^{-1}\left\|w_{j}-c_{i j}\right\|_{L^{2}\left(F_{i j}\right)}^{2} \\
& \leq C h_{i}^{-1}\left\|w_{j}\right\|_{L^{2}\left(F_{i j}\right)}^{2} \\
& \leq C h_{i}^{-1} \delta\left(1+\log \left(H_{j} / \delta\right)\right)\left\|w_{j}\right\|_{H^{1 / 2}\left(F_{j}\right)}^{2} \\
& \leq C\left(1+\log \left(H_{i} / h_{i}\right)\right)\left|w_{j}\right|_{H^{1 / 2}\left(\partial \Omega_{j}\right)}^{2} .
\end{aligned}
$$

Copyright $@$ by SIAM. Unauthorized reproduction of this article is prohibited. 
Here we have used an inverse inequality, the stability of $\pi_{F}$ and $Q$ in the $L^{2}$-norm, the inequality

$$
\left\|c_{i j}\right\|_{L^{2}\left(F_{i j}\right)}^{2} \leq C\left\|w_{j}\right\|_{L^{2}\left(F_{i j}\right)}^{2},
$$

Lemma 3.4 of Dryja and Widlund [7] for the fourth inequality, the Poincaré inequality in the last inequality, and that $\delta$ is comparable to the mesh size $h_{i}$. We note that we have only one log factor in this case.

Therefore, (4.6) combined with (4.7) and (4.9) or (4.10) proves the desired bound

With the help of Lemma 4.5, we can establish property (P2) for the operator $E_{D}$.

Lemma 4.6. With Assumptions 4.2 and 4.3 , the operator $E_{D}$ satisfies

$$
\left|E_{D} w\right|_{\widetilde{S}}^{2} \leq C \max _{i}\left\{\left(1+\log \frac{H_{i}}{h_{i}}\right)^{2}\right\}|w|_{\widetilde{S}}^{2} \quad \text { for any } w \in \widetilde{W}
$$

where $\widetilde{S}$ is defined in (2.11).

Proof. Using the weight matrix $D$ of (3.4), the average operator $E_{D}$, given by (4.1), satisfies

$$
E_{D}\left(\begin{array}{c}
w_{n} \\
w_{m} \\
w_{\Pi}
\end{array}\right)=\left(\begin{array}{c}
w_{n}-B_{n}^{-1}\left(B_{n} w_{n}+B_{m} w_{m}+B_{\Pi} w_{\Pi}\right) \\
w_{m} \\
w_{\Pi}
\end{array}\right),
$$

as in Remark 4.1. Here $w=\left(w_{n}, w_{m}, w_{\Pi}\right) \in \widetilde{W}$. Let

$$
\widehat{w}_{n}=w_{n}-B_{n}^{-1}\left(B_{n} w_{n}+B_{m} w_{m}+B_{\Pi} w_{\Pi}\right),
$$

and construct $\widehat{w}_{i}$ by restricting the unknowns $\left(\widehat{w}_{n}, w_{m}, w_{\Pi}\right)$ to the subdomain $\Omega_{i}$. Similarly, we construct $w_{i}$ from $\left(w_{n}, w_{m}, w_{\Pi}\right)$. We note that $\left(w_{1}, \ldots, w_{N}\right)$ satisfies the primal constraints on the edges/faces. By definition, $\widehat{w}=\left(\widehat{w}_{1}, \ldots, \widehat{w}_{N}\right) \in \widehat{W}$; i.e., $\widehat{w}$ satisfies all of the mortar matching conditions, and each $\widehat{w}_{i}$ is of the form

$$
\widehat{w}_{i}=w_{i}-\sum_{F \subset \partial \Omega_{i}} \tilde{\pi}_{F}\left(w_{i}-\phi\right),
$$

where $F$ is a nonmortar edge/face of $\partial \Omega_{i}, \widetilde{\pi}_{F}\left(w_{i}-\phi\right)$ is the zero extension of $\pi_{F}\left(w_{i}-\phi\right)$ to all of $\partial \Omega_{i} \backslash F$, and $\phi=w_{j}$ on $F_{i j}:=\partial \Omega_{j} \cap \partial \Omega_{i} \subset F$. We then obtain

$$
\begin{aligned}
\left|E_{D} w\right|_{\widetilde{S}}^{2} & =\sum_{i=1}^{N}\left\langle S^{(i)} \widehat{w}_{i}, \widehat{w}_{i}\right\rangle \\
& \leq C \sum_{i=1}^{N}\left(\left\langle S^{(i)} w_{i}, w_{i}\right\rangle+\sum_{F \subset \partial \Omega_{i}}\left\langle S^{(i)} \widetilde{\pi}_{F}\left(\phi-w_{i}\right), \widetilde{\pi}_{F}\left(\phi-w_{i}\right)\right\rangle\right) \\
& \leq C\left(\sum_{i=1}^{N}\left\langle S^{(i)} w_{i}, w_{i}\right\rangle+\sum_{i=1}^{N} \sum_{F \subset \partial \Omega_{i}} \rho_{i}\left\|\pi_{F}\left(\phi-w_{i}\right)\right\|_{H_{00}^{1 / 2}(F)}^{2}\right) \\
& \leq C \max _{i}\left\{\left(1+\log \frac{H_{i}}{h_{i}}\right)^{2}\right\} \sum_{i=1}^{N}\left\langle S^{(i)} w_{i}, w_{i}\right\rangle \\
& =C \max _{i}\left\{\left(1+\log \frac{H_{i}}{h_{i}}\right)^{2}\right\}\langle\widetilde{S} w, w\rangle .
\end{aligned}
$$

Copyright $\odot$ by SIAM. Unauthorized reproduction of this article is prohibited. 
Here we have used that $\left\langle S^{(i)} w_{i}, w_{i}\right\rangle \simeq \rho_{i}\left|w_{i}\right|_{H^{1 / 2}\left(\partial \Omega_{i}\right)}^{2}$, the bounds in (4.2), and Lemma 4.5.

By using the properties (P1) and (P2), we can show the following condition number bound for the BDDC operator (3.6). A proof for a quite similar case is given in Li and Widlund [22] in their analysis of a BDDC method for the Stokes problem with conforming meshes. We do not include a proof, which would be almost identical to that of $[22]$.

THEOREM 4.7. With Assumptions 4.2 and 4.3, we have the condition number bound

$$
\kappa\left(B_{D D C}\right) \leq C \max _{i}\left\{\left(1+\log \frac{H_{i}}{h_{i}}\right)^{2}\right\}
$$

Remark 4.8. For a geometrically nonconforming partition, the number of primal constraints tends to be larger than for a conforming partition if only edge/face constraints are used. We note that there are several previous studies which explore the possibility of selecting primal constraints for only some of the edges/faces; see $[15,21,19]$.

5. A connection between the FETI-DP and BDDC methods. In this section, we will show that the BDDC method developed in the previous sections is closely connected to the FETI-DP method developed by the first author in $[14,15]$ and jointly with Lee in [12]. We will show that the two methods share the same spectra except possibly for an eigenvalue equal to 1.

As previously noted, a comparison of the spectra of the BDDC method to that of the FETI-DP method was made by Mandel, Dohrmann, and Tezaur [25] for conforming finite elements. They showed that the two algorithms have the same set of eigenvalues except possibly for eigenvalues equal to 1 . A simpler proof of this fact was given more recently by $\mathrm{Li}$ and Widlund [23]. They formulated the BDDC operators, as well as the FETI-DP operators, using a change of variables and introducing certain projections and average operators. These projections and average operators provide an important connection between the FETI-DP and the BDDC operators.

We now formulate an FETI-DP operator after the same change of variables as in section 2.2. We then show that the FETI-DP operator has essentially the same spectrum as the BDDC operator by establishing several properties of the projections and average operators that were used by Li and Widlund [23].

After the change of variables, the linear system considered in the FETI-DP formulation is given by

$$
\left(\begin{array}{ccc}
S_{\Delta \Delta} & S_{\Delta \Pi} & B_{\Delta}^{t} \\
S_{\Pi \Delta} & S_{\Pi \Pi} & B_{\Pi}^{t} \\
B_{\Delta} & B_{\Pi} & 0
\end{array}\right)\left(\begin{array}{c}
u_{\Delta} \\
u_{\Pi} \\
\lambda
\end{array}\right)=\left(\begin{array}{c}
g_{\Delta} \\
g_{\Pi} \\
0
\end{array}\right),
$$

where the matrices $S_{\Delta \Delta}, S_{\Delta \Pi}, S_{\Pi \Delta}$, and $S_{\Pi \Pi}$ are defined in (2.12) and the matrices $B_{\Delta}$ and $B_{\Pi}$ are obtained from the mortar matching condition (2.9). We recall that the subscripts $\Pi$ and $\Delta$ stand for the unknowns or submatrices related to the primal variables and the remaining part, respectively, and that $\lambda \in \bar{M}$, the reduced, nonredundant Lagrange multiplier space.

After eliminating the unknowns $u_{\Delta}$ and $u_{\Pi}$, we obtain an equation for $\lambda \in \bar{M}$ :

$$
B_{\Gamma} \widetilde{S}^{-1} B_{\Gamma}^{t} \lambda=d
$$


where

$$
B_{\Gamma}=\left(\begin{array}{ll}
B_{\Delta} & B_{\Pi}
\end{array}\right), \quad \widetilde{S}=\left(\begin{array}{ll}
S_{\Delta \Delta} & S_{\Delta \Pi} \\
S_{\Pi \Delta} & S_{\Pi \Pi}
\end{array}\right),
$$

and $d$ is also the result of the Gaussian elimination.

We will now express the Neumann-Dirichlet preconditioner considered in $[14,15$, 12] using the new unknowns. The Neumann-Dirichlet preconditioner $M_{D P}^{-1}$ is defined by

$$
\left\langle M_{D P} \lambda, \lambda\right\rangle=\max _{w_{\Delta, n} \in W_{\Delta, n}} \frac{\left\langle B_{\Gamma} \mathcal{E}\left(w_{\Delta, n}\right), \lambda\right\rangle^{2}}{\left\langle\widetilde{S} \mathcal{E}\left(w_{\Delta, n}\right), \mathcal{E}\left(w_{\Delta, n}\right)\right\rangle},
$$

where $\mathcal{E}\left(w_{\Delta, n}\right)$ is the extension by zero of $w_{\Delta, n} \in W_{\Delta, n}$ to elements in the space $\widetilde{W}=W_{\Delta, n} \oplus W_{\Delta, m} \oplus \widehat{W}_{\Pi}$.

We recall that the matrix $B_{\Delta}$ is partitioned into

$$
B_{\Delta}=\left(\begin{array}{ll}
B_{n} & B_{m}
\end{array}\right),
$$

where $n$ denotes the columns of the nonmortar unknowns and $m$ those that remain. The formula (5.4) can then be written as

$$
\left\langle M_{D P} \lambda, \lambda\right\rangle=\max _{w_{\Delta, n} \in W_{\Delta, n}} \frac{\left\langle B_{n} w_{\Delta, n}, \lambda\right\rangle^{2}}{\left\langle S_{n n} w_{\Delta, n}, w_{\Delta, n}\right\rangle},
$$

where $S_{n n}$ is the submatrix of $S_{\Delta \Delta}$ in (5.1) corresponding to the nonmortar part. We see that $S_{n n}: W_{\Delta, n} \rightarrow W_{\Delta, n}^{\prime}$ and $B_{n}^{t}: \bar{M} \rightarrow W_{\Delta, n}^{\prime}$ are invertible. Here $W_{\Delta, n}^{\prime}$ is the space dual to $W_{\Delta, n}$. The maximum in (5.5) occurs when $S_{n n} w_{\Delta, n}=B_{n}^{t} \lambda$, and hence it follows that

$$
M_{D P}^{-1}=\left(B_{n}^{t}\right)^{-1} S_{n n} B_{n}^{-1} .
$$

Furthermore, this matrix can be written as

$$
M_{D P}^{-1}=B_{\Sigma, \Gamma} \widetilde{S} B_{\Sigma, \Gamma}^{t},
$$

where

$$
B_{\Sigma, \Gamma}^{t}=\left(\begin{array}{ccc}
\Sigma_{n n} & & \\
& \Sigma_{m m} & \\
& & \Sigma_{\Pi \Pi}
\end{array}\right)\left(\begin{array}{c}
B_{n}^{t} \\
B_{m}^{t} \\
B_{\Pi}^{t}
\end{array}\right)
$$

with the weights given by

$$
\Sigma_{n n}=\left(B_{n}^{t} B_{n}\right)^{-1}, \quad \Sigma_{m m}=0, \quad \Sigma_{\Pi \Pi}=0 .
$$

Therefore, the FETI-DP operator with the Neumann-Dirichlet preconditioner $M_{D P}^{-1}$ is given by

$$
M_{D P}^{-1} F_{D P}=B_{\Sigma, \Gamma} \widetilde{S} B_{\Sigma, \Gamma}^{t} B_{\Gamma} \widetilde{S}^{-1} B_{\Gamma}^{t},
$$

while the preconditioned BDDC operator is given by

$$
B_{D D C}=R_{D, \Gamma}^{t} \widetilde{S}^{-1} R_{D, \Gamma} R_{\Gamma}^{t} \widetilde{S} R_{\Gamma} .
$$

Let us now define the following jump and average operators:

$$
P_{\Sigma}=B_{\Sigma, \Gamma}^{t} B_{\Gamma}, \quad E_{D}=R_{\Gamma} R_{D, \Gamma}^{t} .
$$

The following results are provided in [23, section 5].

TheOREM 5.1. Assume that $P_{\Sigma}$ and $E_{D}$ satisfy 
1. $E_{D}+P_{\Sigma}=I$,

2. $E_{D}^{2}=E_{D}, P_{\Sigma}^{2}=P_{\Sigma}$, and

3. $E_{D} P_{\Sigma}=P_{\Sigma} E_{D}=0$.

Then the operators $M_{D P}^{-1} F_{D P}$ and $B_{D D C}$ have the same eigenvalues except possibly for an eigenvalue equal to 1.

We will now show that the assumptions of Theorem 5.1 hold for the operators $P_{\Sigma}$ and $E_{D}$. We recall the definition of the space of functions satisfying the primal constraints

$$
\widetilde{W}=\left\{\left(w_{n}^{t}, w_{m}^{t}, w_{\Pi}^{t}\right)^{t}: \forall w_{n} \in W_{\Delta, n}, w_{m} \in W_{\Delta, m}, w_{\Pi} \in \widehat{W}_{\Pi}\right\},
$$

and the mortar finite element space

$$
\widehat{W}=\left\{w \in \widetilde{W}: B_{m} w_{m}+B_{\Pi} w_{\Pi}+B_{n} w_{n}=0\right\} .
$$

We note that $P_{\Sigma}$ and $E_{D}$ are operators defined on the space $\widetilde{W}$.

Lemma 5.2. The operators $P_{\Sigma}$ and $E_{D}$ satisfy the assumptions of Theorem 5.1.

Proof. From

$$
\begin{aligned}
& \Sigma_{m m}=0, \quad \Sigma_{\Pi \Pi}=0, \quad \Sigma_{n n}=\left(B_{n}^{t} B_{n}\right)^{-1} \\
& D_{m m}=I, \quad D_{\Pi \Pi}=I, \quad D_{n n}=0,
\end{aligned}
$$

we have

$$
\begin{aligned}
& P_{\Sigma} w=\left(\begin{array}{c}
B_{n}^{-1}\left(B_{m} w_{m}+B_{\Pi} w_{\Pi}+B_{n} w_{n}\right) \\
0 \\
0
\end{array}\right), \\
& E_{D} w=\left(\begin{array}{c}
-B_{n}^{-1}\left(B_{m} w_{m}+B_{\Pi} w_{\Pi}\right) \\
w_{m} \\
w_{\Pi}
\end{array}\right) .
\end{aligned}
$$

Hence,

$$
E_{D}+P_{\Sigma}=I .
$$

From $E_{D} w=w$ and $P_{\Sigma} w=0$ for all $w \in \widehat{W}$, and from $\operatorname{Range}\left(E_{D}\right) \subset \widehat{W}$, we obtain

$$
E_{D}^{2}=E_{D}, \quad P_{\Sigma} E_{D}=0
$$

From (5.7), we have the identities

$$
E_{D}\left(E_{D}+P_{\Sigma}\right)=E_{D}, \quad P_{\Sigma}\left(E_{D}+P_{\Sigma}\right)=P_{\Sigma}
$$

and combining them with (5.8), we obtain

$$
E_{D} P_{\Sigma}=0, \quad P_{\Sigma}^{2}=P_{\Sigma} .
$$

Remark 5.3. Other FETI-DP preconditioners in two dimensions with different weights

$$
\Sigma=\left(\begin{array}{ccc}
\Sigma_{n n} & & \\
& \Sigma_{m m} & \\
& & \Sigma_{\Pi \Pi}
\end{array}\right),
$$

Copyright (c) by SIAM. Unauthorized reproduction of this article is prohibited. 
with nonzero weights $\Sigma_{m m}$ and $\Sigma_{\Pi \Pi}$, have been developed and shown to give condition number bounds of the form

$$
C \max _{i}\left\{\left(1+\log \left(H_{i} / h_{i}\right)\right)^{2}\right\}
$$

for geometrically conforming partitions; see $[8,6]$. We have not found any weight matrix $D$ that results in $E_{D}+P_{\Sigma}=I$ for such a choice of $\Sigma$.

6. Numerical results. In this section, we present numerical results. We first compare the BDDC and the FETI-DP methods with the suggested preconditioners, for geometrically conforming cases, and we then illustrate the performance of our BDDC methods for some geometrically nonconforming partitions. We solve an elliptic problem with the exact solution $u(x, y)=\sin (\pi x)(1-y) y$,

$$
\begin{array}{r}
-\Delta u=f \text { in } \Omega, \\
u=0 \text { on } \partial \Omega,
\end{array}
$$

where $\Omega$ is the unit square in $\mathbb{R}^{2}$. The conjugate gradient iteration is halted when the $\ell_{2}$-norm of the relative residual has been reduced by a factor of $10^{6}$.

In the first series of experiments, the domain $\Omega$ is divided into uniform square subdomains, as in Figure 2, that are geometrically conforming. Common values at the subdomain vertices are selected as the primal constraints for this case. Each subdomain has either a nonuniform mesh or a uniform mesh with $n$ nodes on each subdomain edge. The meshes do not match and have comparable mesh sizes across the interface as in Figure 2.

In Table 1, we show the performance of the two algorithms when $\Omega$ is partitioned into $N=4 \times 4$ subdomains (see Figure 2) and with the local problem size $n$ increasing. In this case, the upper and the right edges of each subdomain are selected to be nonmortar edges; see Figure 2. We provide the $L^{2}$ - and $H^{1}$-errors between the exact solution and the solution of the iterative method, the number of conjugate gradient iterations, and the minimum and the maximum eigenvalues of the BDDC and the FETI-DP methods. For the $H^{1}$-error, we use the broken $H^{1}$-norm given by the subdomain partition. Table 2 shows the numerical results when we fix the local problem size to $n-1=4$ and increase $N$, the number of subdomains to $N=8 \times 8$, $16 \times 16$, and $32 \times 32$, and divide $\Omega$ into square subdomains in the same manner as for $N=4 \times 4$. We observe that the two methods give the same $L^{2}$ - and $H^{1}$-errors. The minimum eigenvalue of the BDDC operator is always equal to 1 while that of the
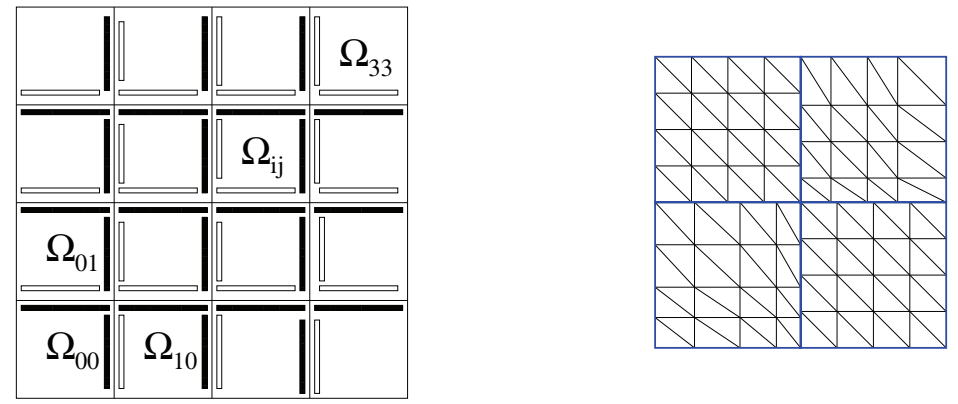

FIG. 2. A subdomain partition (left: white edges are mortar and black edges are nonmortar) with $N=4 \times 4$ and nonmatching comparable meshes with the local problem size $n-1=4$. 
TABLE 1

Comparison of FETI-DP and BDDC methods where $n$, the local problem size, increases with a fixed subdomain partition $(N=4 \times 4)$.

\begin{tabular}{|c||c|c||c|c|c||c|c|c|}
\hline \multicolumn{1}{|c||}{} & \multirow{2}{*}{\multicolumn{1}{|c||}{}} & \multicolumn{1}{c||}{$M_{D P}^{-1} F_{D P}$} & \multicolumn{3}{c|}{$B_{D D C}$} \\
\cline { 4 - 9 }$n-1$ & $\left\|u-u^{h}\right\|_{0}$ & $\left\|u-u^{h}\right\|_{1}$ & Iter & $\lambda_{\min }$ & $\lambda_{\max }$ & Iter & $\lambda_{\min }$ & $\lambda_{\max }$ \\
\hline 4 & $5.0850 \mathrm{e}-4$ & $6.0126 \mathrm{e}-2$ & 10 & 1.40 & 4.09 & 12 & 1.00 & 4.09 \\
8 & $1.2865 \mathrm{e}-4$ & $3.0128 \mathrm{e}-2$ & 13 & 1.01 & 5.72 & 15 & 1.00 & 5.72 \\
16 & $3.2231 \mathrm{e}-5$ & $1.5072 \mathrm{e}-2$ & 15 & 1.00 & 7.72 & 16 & 1.00 & 7.72 \\
32 & $8.0621 \mathrm{e}-6$ & $7.5374 \mathrm{e}-3$ & 16 & 1.01 & $1.00 \mathrm{e}+1$ & 17 & 1.00 & $1.00 \mathrm{e}+1$ \\
64 & $2.0134 \mathrm{e}-6$ & $3.7688 \mathrm{e}-3$ & 17 & 1.01 & $1.28 \mathrm{e}+1$ & 19 & 1.00 & $1.28 \mathrm{e}+1$ \\
\hline
\end{tabular}

TABLE 2

Comparison of FETI-DP and BDDC methods when $N$, the number of subdomains, increases with a fixed local problem size $(n-1=4)$.

\begin{tabular}{|c||c|c||c|c|c||c|c|c|}
\hline \multicolumn{1}{|c||}{} & \multirow{2}{*}{\multicolumn{1}{|c||}{}} & \multicolumn{1}{c||}{$M_{D P}^{-1} F_{D P}$} & \multicolumn{3}{c||}{$B_{D D C}$} \\
\cline { 4 - 9 }$N$ & $\left\|u-u^{h}\right\|_{0}$ & $\left\|u-u^{h}\right\|_{1}$ & Iter & $\lambda_{\min }$ & $\lambda_{\max }$ & Iter & $\lambda_{\min }$ & $\lambda_{\max }$ \\
\hline $4 \times 4$ & $5.0850 \mathrm{e}-4$ & $6.0126 \mathrm{e}-2$ & 10 & 1.40 & 4.09 & 12 & 1.00 & 4.09 \\
$8 \times 8$ & $1.1744 \mathrm{e}-4$ & $2.9900 \mathrm{e}-2$ & 11 & 1.37 & 4.41 & 12 & 1.00 & 4.41 \\
$16 \times 16$ & $2.9743 \mathrm{e}-5$ & $1.4980 \mathrm{e}-2$ & 12 & 1.32 & 4.49 & 13 & 1.00 & 4.49 \\
$32 \times 32$ & $7.4317 \mathrm{e}-6$ & $7.4917 \mathrm{e}-3$ & 12 & 1.30 & 4.57 & 13 & 1.00 & 4.62 \\
\hline
\end{tabular}

FETI-DP operator is greater than 1. The maximum eigenvalues of both operators are almost the same; the eigenvalues are estimated by using the parameters of the conjugate gradient iteration. We note that the minimum eigenvalue of the FETI-DP operator converges to 1 when the number of nodes increases; see Table 1 . The two algorithms perform quite similarly with good scalability in terms of the local problem size and the number of subdomains.

We next illustrate the performance of the BDDC method for geometrically nonconforming partitions. We divide the unit square $\Omega$ into rectangular subdomains that are geometrically nonconforming. For a given $N$, we first divide $\Omega$ into $N$ uniform vertical strips and then each strip into $N$ or $N+1$ rectangles, in succession; see Figure 3 for $N=4$. Each subdomain has a uniform mesh with a number of nodes across the subdomain equal to $n, n+2$, or $n+4$; see Figure 3 . We consider the case when the coefficient $\rho(x)=1$ in $\Omega$ and the case when the coefficient $\rho(x)$ has jumps across the subdomain interfaces; i.e., $\rho(x)=\rho_{i}$, with different constants in different subdomains $\Omega_{i}$. See Figure 3 for the distribution of the $\rho_{i}$ with the values 1,10,100, and 1000 in a partition with $N=4$, and for the selection of nonmortar and mortar edges which satisfies Assumption 4.2 with $C$ less than 1. For the uniform case with $\rho(x)=1$, we use the same selection of nonmortar and mortar edges. For a larger $N$, we copy the same pattern periodically. We run the BDDC method with increasing numbers of nodes in a fixed subdomain partition and with an increase of the number of subdomains with a fixed local problem size.

Table 3 presents the condition numbers and the number of iterations for both continuous and discontinuous $\rho(x)$. Since the subdomain partitions are geometrically nonconforming, we have chosen

$$
\int_{F_{i j}}\left(v_{i}-v_{j}\right) \lambda_{i j} d s=0
$$

as the primal constraints for each face $F_{i j}=\partial \Omega_{i} \cap \partial \Omega_{j}$. Here $\lambda_{i j}$ is the sum of the Lagrange multiplier basis functions that are supported in $\bar{F}_{i j}$. We observe good scalability in terms of the number of subdomains and the local problem size for both 


\begin{tabular}{|c|c|c|c|}
\hline \multirow{2}{*}{$\begin{array}{r}n+4 \\
(10) \\
\end{array}$} & $\mathrm{n} \quad(100)$ & \multirow{2}{*}{$\begin{array}{r}n+4 \\
(10)\end{array}$} & n (100) \\
\hline & $n+2$ & & \multirow{2}{*}{$\begin{array}{l}\mathrm{n}+2 \\
(1000)\end{array}$} \\
\hline \multirow{2}{*}{$\begin{array}{c}\mathrm{n}+2 \\
(1)\end{array}$} & (1000) & \multirow{2}{*}{$\begin{array}{c}\mathrm{n}+2 \\
(1)\end{array}$} & \\
\hline & $n+4$ & & $n+4$ \\
\hline \multirow{2}{*}{$\begin{array}{r}n+4 \\
(10)\end{array}$} & (100) & \multirow{2}{*}{$\begin{array}{c}n+4 \\
(10) \\
\end{array}$} & (100) \\
\hline & \multirow{2}{*}{$\begin{array}{c}\mathrm{n}+2 \\
(1000)\end{array}$} & & $n+2$ \\
\hline \multirow{2}{*}{$\begin{array}{c}\mathrm{n}+2 \\
(1)\end{array}$} & & \multirow{2}{*}{$\begin{array}{c}\mathrm{n}+2 \\
(1)\end{array}$} & $(1000)$ \\
\hline & $\mathrm{n}(100)$ & & $\mathrm{n}(100)$ \\
\hline
\end{tabular}

FIG. 3. A geometrically nonconforming partition with $N=4$ and the number of nodes for each subdomain edge for a given $n$, and the values of $\rho_{i}$ (in parentheses) in the jump coefficient case: nonmortar edges (black) and mortar edges (white) which satisfy Assumption 4.2 for the given $\rho_{i}$ with $C$ less than 1.

TABLE 3

Performance of the BDDC algorithm with an increase of $N$ with a fixed local problem size $(n=6)$ and with an increase of the local problem size, $n$, in a geometrically nonconforming partition with $N=4$. Cond (the condition number) and Iter (the number of iterations) are provided.

\begin{tabular}{|c|c|c||c|c|c||c|c|c||c||c|c|}
\hline \multicolumn{9}{|c||}{$\rho(x)=1$} & \multicolumn{6}{c|}{ Jump coefficient $\rho_{i}$} \\
\hline$N$ & Cond & Iter & $n$ & Cond & Iter & $N$ & Cond & Iter & $n$ & Cond & Iter \\
\hline 16 & 12.36 & 23 & 6 & 11.57 & 20 & 16 & 6.68 & 15 & 6 & 6.67 & 14 \\
32 & 12.37 & 24 & 12 & 14.85 & 22 & 32 & 6.68 & 15 & 12 & 7.94 & 15 \\
48 & 12.40 & 24 & 24 & 18.54 & 23 & 48 & 6.68 & 15 & 24 & 9.52 & 17 \\
64 & 12.41 & 24 & 48 & 22.69 & 26 & 64 & 6.69 & 15 & 48 & 11.37 & 18 \\
\hline
\end{tabular}

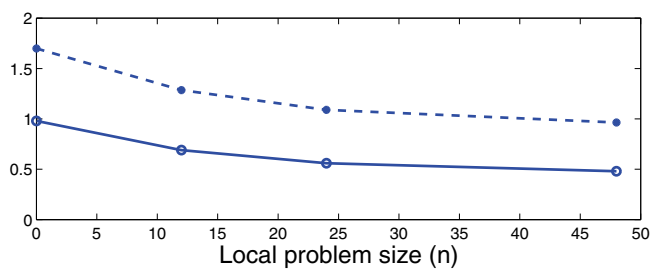

FIG. 4. Plot of the values, Cond $/(1+\log n)^{2}$, with an increase of the local problem size, $n$, in a fixed geometrically nonconforming subdomain partition with $N=4$; the dashed line is for the case $\rho(x)=1$ and the solid line for the case with a jump coefficient $\rho_{i}$.

cases. In addition, the behavior of the condition number with an increase of the local problem size shows that the condition number bound $(1+\log (H / h))^{2}$ appears to be optimal; see Figure 4.

\section{REFERENCES}

[1] Y. Achdou, Y. Maday, And O. B. Widlund, Iterative substructuring preconditioners for mortar element methods in two dimensions, SIAM J. Numer. Anal., 36 (1999), pp. 551580.

[2] F. Ben Belgacem and Y. Maday, The mortar element method for three-dimensional finite elements, RAIRO Modél. Math. Anal. Numér., 31 (1997), pp. 289-302.

[3] C. Bernardi, Y. Maday, and A. T. Patera, A new nonconforming approach to domain decomposition: The mortar element method, in Nonlinear Partial Differential Equations and their Applications. Collège de France Seminar, Vol. XI (Paris, 1989-1991), Pitman

Copyright (C) by SIAM. Unauthorized reproduction of this article is prohibited. 
Res. Notes Math. Ser. 299, Longman Sci. Tech., Harlow, UK, 1994, pp. 13-51.

[4] D. Braess, Finite Elements, in Theory, fast solvers, and applications in solid mechanics, Translated from the 1992 German original by Larry L. Schumaker, Cambridge University Press, Cambridge, 1997.

[5] C. R. Dohrmann, A preconditioner for substructuring based on constrained energy minimization, SIAM J. Sci. Comput., 25 (2003), pp. 246-258.

[6] N. Dokeva, M. Dryja, And W. Proskurowski, A FETI-DP preconditioner with special scaling for mortar discretization of elliptic problems with discontinuous coefficients, SIAM J. Numer. Anal., 44 (2006), pp. 283-299.

[7] M. Dryja And O. B. WidLund, Domain decomposition algorithms with small overlap, SIAM J. Sci. Comput., 15 (1994), pp. 604-620.

[8] M. DRYja and O. B. WidLund, A generalized FETI-DP method for a mortar discretization of elliptic problems, in Domain Decomposition Methods in Science and Engineering, I. Herrera, D. E. Keyes, O. B. Widlund, and R. Yates, eds., National Auton. Universidad de Mexico, México, 2003, pp. 27-38.

[9] C. Farhat, M. Lesoinne, and K. Pierson, A scalable dual-primal domain decomposition method, Numer. Linear Algebra Appl., 7 (2000), pp. 687-714.

[10] P. Grisvard, Elliptic Problems in Nonsmooth Domains, in Monographs and Studies in Mathematics 24, Pitman (Advanced Publishing Program), Boston, 1985.

[11] C. Kim, R. D. Lazarov, J. E. Pasciak, and P. S. Vassilevski, Multiplier spaces for the mortar finite element method in three dimensions, SIAM J. Numer. Anal., 39 (2001), pp. 519-538.

[12] H. H. Kim AND C.-O. LeE, A preconditioner for the FETI-DP formulation with mortar methods in two dimensions, SIAM J. Numer. Anal., 42 (2005), pp. 2159-2175.

[13] H. H. Kim And O. B. WidLund, Two-level Schwarz algorithms with overlapping subregions for mortar finite elements, SIAM J. Numer. Anal., 44 (2006), pp. 1514-1534.

[14] H. H. KIM, A FETI-DP preconditioner for mortar methods in three dimensions, Electron. Trans. Numer. Anal., 26 (2007), pp. 103-120.

[15] H. H. KIM, A FETI-DP formulation of three dimensional elasticity problems with mortar discretization, SIAM J. Numer. Anal., 46 (2008), pp. 2346-2370.

[16] A. Klawonn and O. Rheinbach, A parallel implementation of dual-primal FETI methods for three-dimensional linear elasticity using a transformation of basis, SIAM J. Sci. Comput., 28 (2006), pp. 1886-1906.

[17] A. Klawonn and O. Rheinbach, Inexact FETI-DP methods, Internat. J. Numer. Methods Engrg., 69 (2007), pp. 284-307.

[18] A. Klawonn and O. Rheinbach, Robust FETI-DP methods for heterogeneous three dimensional elasticity problems, Comput. Methods Appl. Mech. Engrg., 196 (2007), pp. 14001414 .

[19] A. Klawonn, O. B. Widlund, And M. DRYjA, Dual-primal FETI methods for threedimensional elliptic problems with heterogeneous coefficients, SIAM J. Numer. Anal., 40 (2002), pp. 159-179.

[20] A. Klawonn and O. B. Widlund, Dual and dual-primal FETI methods for elliptic problems with discontinuous coefficients in three dimensions, in Domain Decomposition Methods in Sciences and Engineering (Chiba, 1999), DDM.org, Augsburg, Germany, 2001, pp. 29-39.

[21] A. Klawonn and O. B. WidLund, Dual-primal FETI methods for linear elasticity, Comm. Pure Appl. Math., 59 (2006), pp. 1523-1572.

[22] J. Li AND O. WidLund, BDDC algorithms for incompressible Stokes equations, SIAM J. Numer. Anal., 44 (2006), pp. 2432-2455.

[23] J. Li AND O. B. WidLund, FETI-DP, BDDC, and block Cholesky methods, Internat. J. Numer. Methods Engrg., 66 (2006), pp. 250-271.

[24] J. Li AND O. WidLund, On the use of inexact subdomain solvers for BDDC algorithms, Comput. Methods Appl. Mech. Engrg, 196 (2007), pp. 1415-1428.

[25] J. Mandel, C. R. Dohrmann, and R. Tezaur, An algebraic theory for primal and dual substrucruting methods by constraints, Appl. Numer. Math., 54 (2005), pp. 167-193.

[26] J. Mandel and C. R. Dohrmann, Convergence of a balancing domain decomposition by constraints and energy minimization, Numer. Linear Algebra Appl., 10 (2003), pp. 639-659.

[27] J. Mandel, B. Sousedík, and C. R. Dohrmann, On multilevel BDDC, in Proceedings of the 17th International Conference on Domain Decomposition Methods in Science and Engineering, Strobl, Austria, 2006, U. Langer, M. Discacciati, D. Keyes, O. Widlund, and W. Zulehner, eds., Lect. Notes Comput. Sci. Engrg. 60, Springer-Verlag, Berlin, 2007, pp. 287-294.

[28] A. Toselli And O. Widlund, Domain decomposition methods-Algorithms and theory, 
Springer Ser. Comput. Math. 34, Springer-Verlag, Berlin, 2005.

[29] X. Tu, Three-level BDDC in three dimensions, SIAM J. Sci. Comput., 29 (2007), pp. 17591780.

[30] X. TU, Three-level BDDC in two dimensions, Internat. J. Numer. Methods Engrg., 69 (2007), pp. 33-59.

[31] B. I. Wohlmuth, A mortar finite element method using dual spaces for the Lagrange multiplier, SIAM J. Numer. Anal., 38 (2000), pp. 989-1012.

[32] B. I. Wohlmuth, Discretization Methods and Iterative Solvers Based on Domain Decomposition, Lect. Notes Comput. Sci. Engrg. 17, Springer-Verlag, Berlin, 2001.

Copyright (c) by SIAM. Unauthorized reproduction of this article is prohibited. 